\title{
La compétence métapragmatique chez les enfants de 9 à 11 ans - Processus cognitifs et effets de saillance
}

\author{
Laurence Buson \\ Laboratoire Lidilem - Université Stendhal Grenoble 3 \\ laurence.buson@u-grenoble3.fr
}

La part de conscience inhérente au phénomène de souplesse stylistique nécessite d'explorer en détail les représentations et jugements que les enfants se forgent sur ce champ de variation, de manière à mieux comprendre à la fois les processus cognitifs en jeu dans l'évaluation des styles, et les problématiques socio-identitaires sous-jacentes aux discours sur le style, ces deux dimensions étant susceptibles d'interagir avec les pratiques stylistiques. En effet, les modalités perceptives ainsi que le sens social attribué à la variation et aux variétés peuvent constituer des informations précieuses pour être ensuite en mesure d'interpréter certains usages et comportements langagiers (Schilling-Estes, 2002).

Après un retour théorique sur le processus de construction des représentations et en explorant les notions de saillance et de stéréotypie qui mènent, chez l'adulte comme chez l'enfant, à élaborer un jugement sur les styles, nous nous intéresserons aux étapes d'acquisition de la compétence métapragmatique, avant d'entrer dans le détail d'une étude menée auprès de 196 enfants de 9/11 ans visant à explorer les manifestations de la compétence de réception chez des locuteurs pré-adolescents (Buson, 2009). Les analyses que nous détaillerons ensuite s'attachent à en explorer trois aspects : quelles formes prennent ces représentations ? comment se construisent les représentations sur le style ? et sur quels traits saillants les enfants fondent-ils leurs jugements?

\section{Comment se construisent les représentations du style : entre saillance et stéréotypie}

Le processus de construction des représentations et des jugements sur les styles relève de la compétence de réception qui implique, d'un point de vue cognitif,

\begin{abstract}
the ability to on the one hand categorize speech as such and on the other hand relate lectal categories to social categories. In more precise terms, it involves the step from a linguistic trigger to a social schema, activating stored encyclopaedic knowledge in the broad sense, including ideological aspects and psychological attributes associated with the group in question. (Kristiansen, $2008: 50$ )
\end{abstract}

Elle fait donc appel au processus qui consiste à établir des liens plus ou moins conscients entre les styles et les significations sociales, et par conséquent aux notions de saillance (qui s'appuie sur une modalité ascendante) et de stéréotype (qui s'appuie sur une modalité descendante).

La modalité ascendante (bottom-up) part des plus petites unités pour aller vers des unités plus complexes et plus abstraites, alors que la modalité descendante (top-down) commence par les unités larges et conceptuelles pour finir avec les unités de bas niveau. La question est de savoir comment interagissent les deux sources d'informations et laquelle prédomine éventuellement en cas de conflit cognitif. Nous allons voir ici en quoi ces deux dimensions sont essentielles dans le champ de la représentation de la variation et comment elles interagissent dans la perception et l'évaluation du style.

\subsection{Perception globale descendante et stéréotypes sociaux}

La modalité descendante s'appuierait sur une perception globale de l'énoncé, et partirait du contenu, donc d'une impression générale sur un énoncé structurée en fonction d'un horizon d'attentes. Cet horizon 
d'attentes serait fondé sur un ensemble de représentations préalables, influencées par l'histoire propre de l'individu et par les stéréotypes dont il est porteur, c'est-à-dire des croyances socialement partagées concernant les caractéristiques communes d'un groupe social.

Le locuteur catégorise son interlocuteur, et cherche à se positionner par rapport à lui, sans que l'une ou l'autre des places ne soit figée dans le temps. Le processus de catégorisation qui est ici décrit est à rapprocher de la notion d'iconisation de Irvine \& Gal (Gal \& Irvine, 1995 ; Irvine \& Gal, 2000) et qui consiste en la construction d'une relation nouvelle, ancrée dans l'interaction, entre le style et une image sociale du locuteur, que l'individu concevra comme non arbitraire et sémiotiquement motivée.

Le style étant intrinsèquement hétérogène, l'individu en réception tend à réduire le phénomène de dissonance cognitive provoqué par la non-congruence de certains traits en recréant de l'homogène à partir de l'hétérogène. Les stéréotypes, qui orientent l'attention et l'interprétation vers ce qui est consonant en fonction des attentes, limitent ainsi l'impact des informations discordantes, voire conduisent à reconstruire des informations qui n'ont pas réellement été perçues, par un effet global d'homogénéisation (Louvet, 2005).

Cette notion rappelle celle de gommage (erasure) proposée par Irvine (2001), et qui fait système avec l'iconisation en simplifiant la perception sociolinguistique sur la base des représentations du sujet, dans le but de reconstruire une image mentale cohérente ${ }^{1}$ de l'énoncé qui a été perçu. Le gommage correspond au fait que certains traits saillants sont sélectionnés en priorité par le récepteur au détriment de tout un faisceau de marques moins saillantes qui seraient négligées. Ce processus permettrait au récepteur de se forger une représentation consistante à partir d'un énoncé qui ne l'est pas.

Afin d'aborder cette question du rôle de la saillance perceptive, et d'essayer d'envisager la compétence de réception dans toute sa complexité, nous allons maintenant envisager la modalité inverse à la catégorisation top-down, à savoir la modalité ascendante ou bottom-up.

\subsection{Perception ciblée ascendante et saillance}

L'idée d'un rôle spécifique joué par certains traits linguistiques dans la réception et la production du message conduit directement à interroger la notion de saillance puisqu'il s'agit de positionner les éléments de l'énoncé sur une échelle de saillance, reliée à une échelle de conscience de la valeur de ces variables. En sociolinguistique, poser la question de ce que sont les marqueurs et les stéréotypes au sens labovien du terme revient donc à poser la question des facteurs de saillance qui amènent les locuteurs à identifier certains traits et pas d'autres et à leur attribuer une valeur sociale.

D'un point de vue général, « est saillant ce qui arrive en premier à l'esprit, ce qui capte l'attention »; d'un point de vue linguistique, il s'agit d'un élément qui ressort prioritairement, " au point de prendre une importance cognitive particulière et, par exemple, d'axer sur cet élément la réaction ou la réponse à l'énoncé » (Landragin, 2005: 157). Bates \& MacWhinney (1979) postulent que le poids pragmatique d'une information s'évalue à l'aune de sa saillance et de sa nouveauté. Cette question de la saillance perceptive semble donc essentielle dans la construction des représentations. De même, Lodge (2007) reprend les recherches de Kerswill \& Williams (2002) et de Trudgill (1986) pour souligner l'importance de la notion de saillance dans le processus évaluatif :

Par 'saillance' il [(Trudgill, 1986)] entend le niveau de conscience associée par les locuteurs à des variables particulières. La saillance d'une variante comporte plusieurs éléments - degré de stigmatisation, distance phonétique, neutralisation de contrastes phonologiques, etc. Cette définition comporte une part de circularité (voir Kerswill \& Williams, 2002), mais la notion de 'saillance' reste néanmoins utile. (Lodge, 2007: 119)

Toutefois, comme le notent Kerswill \& Williams (2002), il existe un risque de circularité à la définition de la saillance puisque, si l'on considère par exemple, à la suite de Trudgill (1986), qu'un facteur de saillance consiste dans le fait d'être impliqué dans le processus de changement linguistique, comment savoir si la caractéristique première de la variable est d'être impliquée dans ce changement ou d'être 
saillante ? Selon Kerswill \& Williams, le biais de ce type de démarche est de postuler des causes pour des conséquences, et vice-versa, ce que souligne également Kristiansen :

\author{
If lectal varieties constitute prototype categories, some realizations will be more \\ 'typical' or 'central' or 'better examples' of a given variety than others. (Kristiansen, \\ 2008: 59)
}

Les questions qui se posent sont alors celles de l'émergence de cette saillance, des facteurs qui déterminent la prépondérance de certaines variables sur d'autres, et de la part de saillance intralinguistique et extra-linguistique.

De ce point de vue, un premier ensemble de facteurs peut se regrouper sous l'étiquette de « saillance intrinsèque » (Landragin, 2004), dans la mesure où ils correspondent à la nature même de l'élément. Ainsi, par exemple, un terme constitué d'une combinaison de phonèmes peu fréquente peut apparaître comme saillant dans un énoncé. Un deuxième ensemble de facteurs concerne le contexte linguistique de l'élément, et une mise en relief qui s'opère au cours de l'énonciation. Citons par exemple une prosodie particulière ou encore les aspects sémantiques, comme la récence et la nouveauté dans un discours spécifique. Enfin, on peut évoquer une troisième famille de facteurs, ceux qui ont trait au contexte linguistique mais aussi et peut-être surtout extra-linguistique. Par exemple, au niveau sémantique, un trait peut avoir un ancrage affectif fort pour un individu particulier (serpent, vampire, etc.), ou revêtir une importance toute particulière selon le but de l'échange.

En outre, la question de la saillance s'accompagne d'une réflexion sur l'unité de saillance : l'évaluation d'un style se fait-elle à partir d'un trait unique ou d'un ensemble de traits congruents, et quels sont ces traits ? On peut en effet se demander si un phonème peut constituer un trait saillant stylistiquement, et/ou si le mot seul est une unité plus pertinente.

Cette question de la saillance des variables semble également difficile à dissocier des représentations initiales des individus, de leur rapport à la norme, et de leur familiarité avec les variantes. La saillance n'est pas dissociable de l'individu qui perçoit le message : elle se construit sur l'association entre certains éléments du discours et ses représentations. Si certains traits linguistiques peuvent être saillants « en soi », ils ne le seront pas de la même manière chez tous les locuteurs, qui feront porter leur attention de manière privilégiée sur tel ou tel trait ou groupe de traits, guidés en cela par l'émetteur qui accentuera certains éléments de son message, mais aussi par leur propre histoire.

Il est donc probable que les modalités ascendantes et descendantes interagissent plus qu'elles ne s'opposent, la saillance s'appuyant sur les stéréotypes tout en les renforçant, dans un processus qui s'autoalimente en hélice pour construire du sens social. Fuchs (1999) défend d'ailleurs l'idée que la construction du sens se fonde sur la sélection cognitive d'éléments saillants ou cohérents entre eux, qui vont permettre de structurer la réalité et de s'en faire une représentation globale.

\title{
1.3 Un processus perceptif et évaluatif en hélice
}

Selon Michinov \& Monteil (2003), les traits saillants sont en réalité des indices catégoriels, activateurs de stéréotypes et d'identité sociale. Dans le cadre de la théorie exemplariste, ce processus correspondrait à la récupération, du fait de leur similarité avec l'objet perçu, d'exemplaires stockés en mémoire - ce stockage impliquant des formes linguistiques associées à des informations contextuelles - sachant que cette dimension de similarité n'est pas fixée a priori, et qu'elle est fortement dépendante du contexte, une multitude de facteurs étant susceptible de rendre tel ou tel élément plus ou moins saillant pour un récepteur particulier dans une situation bien spécifique (Smith \& Zarate, 1992). L'accumulation de ces exemplaires conduirait ensuite au développement de la conscience de ce lien entre formes et contextes, comme le suggèrent Foulkes \& Docherty (2006).

Cette approche peut être comparée à la perception métonymique évoquée par Kristiansen, par laquelle un trait unique peut suffire à activer un ensemble de traits correspondant au patron représentationnel d'un style particulier : 
Ce jugement serait ensuite éventuellement amendé par la présence d'autres traits saillants cohérents ou divergents avec le stéréotype conçu initialement pour alors participer à le reconfigurer. CampbellKibler (2009) évoque un processus comparable selon lequel les locuteurs attribueraient consciemment un sens social à certaines variantes mais aussi à d'autres, de manière indirecte et plus inconsciente, en regroupant ainsi certains traits linguistiques dans des stylistic packages.

Ces tensions entre l'homogène des niveaux et la réalité de l'hétérogène stylistique, entre saillance et stéréotype, et entre pratiques attendues et inattendues, sont donc au cœur de la réflexion sur l'évaluation de la variation stylistique par les locuteurs.

Si ces problématiques sont pertinentes pour des locuteurs de tout âge, il convient d'aborder plus spécifiquement la question de la construction des représentations sur le style d'un point de vue développemental.

\section{La compétence métapragmatique chez l'enfant : de la semi- conscience de la variation au savoir-dire sur la variation}

La dimension métacognitive liée à la conscience de la variation stylistique peut être définie comme la maîtrise consciente des règles sociales du langage telle qu'elle peut s'observer dans les commentaires explicites sur les usages (Bates, 1976). Il est essentiel de la prendre en compte si l'on s'intéresse à la dimension stratégique des productions puisque la souplesse stylistique, en tant que capacité à réagir mais aussi à agir sur le monde, suppose une conscience des possibles. De plus, les représentations que le locuteur se construit de lui-même et de lui en relation avec l'autre dans une situation donnée vont fortement influencer les pratiques langagières, à travers ce que le locuteur connaît, perçoit, attend et comprend, de la situation d'interaction.

Comme le font remarquer Levelt, Sinclair et al. (1978), Clark (1978) ou encore Slobin (1978), le degré d'explicitation, donc la mise en mots, est une variable pivot dans la reconnaissance des compétences métalangagières. Le débat autour du caractère précoce ou tardif du développement de la compétence métapragmatique, au-delà de la volonté de la situer à un âge précis, semble, si l'on prend en compte un certain degré d'explicitation, aller dans le sens d'une capacité plutôt tardive, et plutôt postérieure aux premières adaptations aux situations. Dans cette optique, le caractère mécanique des ajustements précéderait largement son pôle stratégique. En effet, comme le note Gombert (1990: 127), un grand nombre de composantes de cette capacité réflexive sur les productions socialement situées ne sont acquises que vers 8 ou 10 ans et les "ajustements précoces constatés dans les situations d'interaction sociale [seraient] de nature non consciente, donc non métalinguistique" (Gombert, 1990: 143).

Si les enfants commencent à avoir une conscience métalinguistique avant 6 ans, il semblerait donc que la conscience métapragmatique lui soit postérieure. En effet, Gombert (1990: 131) reprend les résultats de plusieurs études analysant la perception des échecs communicatifs par des enfants d'âge scolaire qui vont dans le sens d'une perception plus objective des situations chez les enfants les plus âgés (au-delà de 8 ans) qui sont les seuls à fonder leur analyse sur le langage lui-même. Ainsi, avant 9-10 ans, les enfants seraient influencés par ce qu'ils savent du locuteur (ainsi un enfant est moins crédible qu'un adulte, et un adulte réputé stupide moins crédible qu'un adulte "normal"), et s'appuieraient davantage sur ces informations extralinguistiques que sur le message lui-même pour construire leurs jugements.

Les adaptations précoces observées chez l'enfant d'âge préscolaire relèveraient alors plutôt de ce que Gombert appelle des comportements épipragmatiques, correspondant à l'intervention manifeste des connaissances du sujet dans les traitements qu'il opère (comme par exemple les autocorrections). Ils correspondent à une phase au cours de laquelle l'enfant opère une forme de contrôle cognitif sur ses productions, tout en étant incapable de dissocier le langage de la situation de production. 
Avec l'âge et une certaine forme de pression environnementale, les connaissances implicites/ épilinguistiques issues des habiletés linguistiques primaires évolueraient vers un contrôle conscient du langage, étape des comportements métapragmatiques, évolution qui se traduirait principalement par une prise en compte du destinataire. Gombert (1990:157) situe les prémisses de ce processus de conscientisation vers 6-7 ans, et précise qu'il se poursuit durant l'adolescence en fonction, selon lui, de la familiarité de l'enfant avec les liens entre langage et contextes d'émission. L'auteur postule en outre que l'entrée dans l'écrit, ainsi qu'un travail réflexif de nature métalinguistique à l'oral, pourraient être des éléments déclencheurs de cette évolution.

Afin d'explorer plus avant les différentes dimensions abordées jusqu'ici et dans le but d'éclairer par des exemples concrets les hypothèses théoriques formulées précédemment, nous allons maintenant décrire une enquête que nous avons menée sur le terrain auprès d'un peu moins de 200 enfants francophones âgés de 9 à 11 ans .

\section{Une étude portant sur les représentations de la variation stylistique chez les 9/11 ans. Questions méthodologiques}

\subsection{Le terrain : 6 écoles et 11 classes de CM de l'agglomération grenobloise (Sud-Est de la France), 196 enfants}

Six établissements scolaires ont été sollicités dans le cadre de ce travail, ce qui correspond à onze classes de CM au total. Nous avons contacté des écoles socialement variées, mixtes, favorisées ou défavorisées, en contexte urbain ou rurbain.

Dans le tableau ci-dessous, les 196 sujets enquêtés sont répartis en fonction de l'origine sociale, de l'âge et du genre. Notre échantillon est donc composé de 107 filles et de 89 garçons issus de milieux sociaux variés :

\begin{tabular}{|c|c|c|c|c|c|}
\hline & Défavorisée & Intermédiaire- & Intermédiaire+ & Favorisée $^{2}$ & \\
\hline 9 ans & $8 \mathrm{~F}+6 \mathrm{G}(14)$ & $9 \mathrm{~F}+4 \mathrm{G}(13)$ & $4 \mathrm{~F}+6 \mathrm{G}(10)$ & $11 \mathrm{~F}+5 \mathrm{G}(16)$ & 53 \\
\hline 10 ans & $21 \mathrm{~F}+9 \mathrm{G}(30)$ & $13 \mathrm{~F}+8 \mathrm{G}(21)$ & $9 \mathrm{~F}+17 \mathrm{G}(26)$ & $9 \mathrm{~F}+11 \mathrm{G}(20)$ & 97 \\
\hline 11 ans & $8 \mathrm{~F}+6 \mathrm{G}(14)$ & $6 \mathrm{~F}+5 \mathrm{G}(11)$ & $5 \mathrm{~F}+4 \mathrm{G}(9)$ & $4 \mathrm{~F}+8 \mathrm{G}(12)$ & 46 \\
\hline & 58 & 45 & 45 & 48 & 196 \\
\hline
\end{tabular}

Tableau 1 : Répartition de l'échantillon

\subsection{Le recueil des déclarations spontanées en réaction à un document audio}

Nous avons mené 196 entretiens individuels enregistrés d'une vingtaine de minutes chacun. Chaque enfant est amené à écouter un document déclencheur et à développer un commentaire non dirigé initié par une question ouverte («Qu'est-ce que tu remarques? Qu'est-ce que tu en penses ?») suivie d'une relance unique. Nos résultats s'appuient sur les analyses essentiellement qualitatives des réponses des enfants.

Le document audio déclencheur simule des annonces de répondeur téléphonique. Il est composé de trois messages stylistiquement contrastés, préalablement écrits et ensuite joués par un locuteur adulte :

(01) Extrait 1 (surveillé) : bonjour - je suis désolé je suis momentanémenT absent - mais si vous le souhaitez vous pouvez m(e) laisseR un message avec vos coordonnées et $j(e)$ vous recontacterai ultérieurement - je vous remercie et à bientôt

(02) Extrait 2 (intermédiaire) : bonjour je suis pas là - si vous voulez vous pouvez $\mathrm{m}(\mathrm{e})$ laisser un message et $j(e)$ vous rappellerai - merci a bientôt 
(03) Extrait 3 (non surveillé) : salut les gars* bon j(e) suis pas là désolé - mais lâchez-vous laissez-moi un message et $\mathrm{j}(\mathrm{e})$ vous rappelle allez à plus ciao

Ici, l'enregistrement a été conçu de manière à faire apparaître une plus grande surveillance à tous les niveaux dans l'extrait 1 (phonétique, syntaxe, lexique, pragmatique), surveillance qui s'amenuise au fur et à mesure des extraits pour aboutir à une formulation qui se veut moins surveillée, tant au niveau de l'intonation et de la phonétique, que du lexique et de la syntaxe.

\section{Analyse des déclarations spontanées : critères d'évaluation des styles et processus perceptif chez les 9/11 ans}

\subsection{Les critères utilisés pour parler de la variation stylistique : rôles sociaux, norme, caractéristiques de l'interaction}

Les commentaires spontanés peuvent être catégorisés dans 8 grandes familles, allant de la non-réponse à la réponse évoquant des critères multiples pour interpréter la variation entendue (cf. figure 1).

Réponses spontanées (Q. ouverte) étude 1

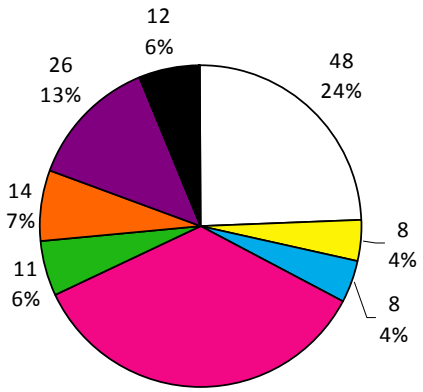

69 $\square$ contenu ou $\varnothing$

¿ social

$\square$ norme

$\square$ inter $^{\circ}$

$\square$ norme\&social

$\square$ inter ${ }^{\circ} \&$ social

$\square$ inter ${ }^{\circ}$ \&norme

- 3 critères

Figure 1 : Types de réponses obtenues à la question ouverte

a) 48 enfants, soit près d'un quart de l'échantillon, n'ont fait ni référence à l'interaction, ni à la norme, ni aux caractéristiques sociales du locuteur. Ces enfants ont été regroupés dans une même catégorie (en blanc sur la figure 1), sous l'étiquette « centration sur le contenu ou non-réponse » :

- certains ne répondent rien ou disent ne pas savoir ;

- certains s'attachent au contenu des messages uniquement :

(04) il y a un monsieur il dit qu'il est absent et après il dit de laisser un message (Mickaël, 9 ans, inter-)

- d'autres semblent percevoir de la variation mais sans réussir à expliciter le propos ou à lui attribuer une valeur sociolinguistique :

(05) en fait c'est une messagerie, ils veulent l'appeler et ils tombent sur son répondeur et il dit "laissez-moi un message". il y en a un où il a répondu "salut les gars", les autres il était sur la messagerie (Laura, 9 ans, défav)

b) 8 enfants, soit $4 \%$ de l'échantillon, font référence aux caractéristiques sociales du locuteur, et ne font référence à aucun autre critère (en jaune sur la figure 1) :

- Nicolas s'identifie à un style (le moins surveillé) qui semble le caractériser dans toutes les situations : 
(06) c'est pas de la même catégorie, c'est pas pareil, la 3 elle est pas pareil que la 1ère. l'accent, enfin pas l'accent, comment il parle. la dernière j'aime bien, c'est plus de mon style, ce que je dis (Nicolas, 10 ans, inter + ).

- Baptiste attribue la variation à l'âge du locuteur :

(07) le 1er on dirait qu'il est plus vieux et puis le 2ème encore un petit peu plus jeune, et le 3ème aussi, c'est encore plus jeune (Baptiste, 10 ans, inter + ).

- Thomas attribue le style surveillé à un locuteur de statut élevé :

(08) le 1er c'est comme si il était docteur (Thomas, 9 ans, fav).

c) 11 enfants, soit $6 \%$ de l'échantillon, font référence à la fois à la norme et aux caractéristiques sociales du locuteur (en vert sur la figure 1):

- Muhammet, Adem et Jérémie font allusion au parler « racaille», « voyou» ou «jeunes du quartier », ainsi qu'au « bien parler », au « parler correct » et à la politesse :

(09) le 3ème il parle un peu comment dire un peu comme une racaille « lâchez-vous », ça parle un peu mal. le 1er ça parle bien (Muhammet, 10 ans, défav)

(10) c'était tous des répondeurs et que ils avaient pas tous la même voix. le ler il est poli et l'autre il parle voyou (Adem, 9 ans, défav)

(11) c'est quelqu'un qui laisse un répondeur, qu'il est pas là. il y a quelqu'un qui était un jeune, qui parle comme les jeunes du quartier quoi, le ler c'était un monsieur sérieux, et le 2ème je sais pas c'est un peu comme le ler, enfin c'est les deux mélangés. le ler il parle bien français et le jeune il parle pas vraiment correctement (Jérémie, 10 ans, inter + )

- Nassima fait référence au milieu social du locuteur et à son niveau d'études :

(12) il y en a un qui est plus, par exemple qui travaille dans un métier et il parle correctement et tout. si il serait pas pauvre il aurait mieux parlé parce que par exemple lui il serait pas allé loin dans ses études et quelqu'un de riche il serait allé loin (Nassima, 9 ans, inter-)

d) 8 enfants (soit 4\%) font référence à la norme uniquement (en bleu sur la figure 1) :

- Nicolas juge le style moins surveillé d'un point de vue moral :

(13) j'aime pas trop c'est pas joli comme il parle "lâchez-vous" et tout ça (Nicolas, 9 ans, inter-).

- Clément fait référence à la politesse et juge certaines formulations grossières :

(14) le 1er il était poli, très poli, le 2ème moins et le dernier pas. parce que il y a des mots qu'ils disent pas tous «lâchez-vous », «bonjour je ne suis pas là » ça c'est une manière de politesse. l'autre je sais pas c'est plus des gros mots (Clément, 11 ans, inter+).

e) 69 enfants, soit plus d'un tiers de l'échantillon, font référence aux caractéristiques de l'interaction (en rose sur la figure 1), et cette proportion monte à plus de $60 \%$ si l'on prend en compte les enfants qui combinent ce critère avec d'autres (en orange, violet et noir sur la figure 1) :

- Par exemple, Simon prend en compte l'interlocuteur, tout en condamnant certains usages, et en expliquant que le fait d'être adulte contraint le locuteur à certaines pratiques :

(15) par exemple « les gars » c'est pas un mot à dire à des personnes, par exemple tu es un adulte tu vas pas dire ça à ton voisin (Simon, 9 ans, inter + ).

- Karine juge la correction des annonces, attribue certaines façons de parler à des locuteurs stéréotypiques, et se projette parallèlement dans les différents styles en intégrant la situation de communication comme variable pour étayer son jugement :

(16) le dernier il a plutôt parlé comme les adolescents et le 1er il a parlé comme si comme si il était bien élevé, enfin il a parlé normalement, pas avec des mots que les jeunes ils prennent. 
la dernière je parle plutôt comme ça mais des fois je parle comme la 1ère mais je sais pas la 1ère c'était un peu trop correct (Karine, 10 ans, inter-).

- Ryad prend en compte l'intention communicative du locuteur, tout en jugeant certains styles très négativement, tant du point de vue de la norme que du point de vue des locuteurs susceptibles de les utiliser :

(17) au début il voulait faire croire à ses copains qu'il était pas là et après il s'est lâché il a changé d'humeur. au début il parle gentil, après il parle normal, et après il parle vraiment méchant, il fait une voix vraiment grave, il a dit d'un air... il fait celui qui est le plus fort et tout, je sais pas comment dire, on dirait un peu qu'il fait sa racaille (Ryad, 11 ans, inter-).

Ces premières analyses nous permettent de constater que plus de $60 \%$ des enquêtés sont capables de formuler une référence aux caractéristiques de l'interaction de manière spontanée, et de préciser les critères sur lesquels les enfants se fondent pour interpréter la variation qu'ils perçoivent. La question est alors de savoir sur quels éléments ils s'appuient précisément pour élaborer ces critères.

\subsection{Le processus perceptif : comment s'articulent la perception des traits saillants et les représentations stéréotypiques}

Tout d'abord, on constate que $70 \%$ des enfants citent un extrait de l'enregistrement dans leurs commentaires, avec plus ou moins d'exactitude selon les enfants. Par exemple, Eva repère certains éléments lexicaux, tant dans le style surveillé que non surveillé, et s'appuie sur ce relevé pour expliquer la variation entre les extraits :

(18) il dit chaque fois la même chose mais chaque fois différemment. chaque fois il montre autrement qu'il est absent, à un moment il dit qu'il est «momentanément absent ", à un autre il dit « je suis pas là » et le 3ème je crois qu'il dit « je suis pas là » aussi. ça dépend à qui tu parles parce que si tu dis «salut les gars » c'est que tu les connais bien, à des amis, sinon si tu dis «bonjour je suis momentanément absent» c'est plutôt à des gens que tu connais pas très bien, style des chefs d'entreprise ou des gens, parce que des fois ils disent des mots assez difficiles (Eva, 9 ans, fav).

Eva parle donc des mots «difficiles» qu'elle a repérés, Antoine, quant à lui, utilise le terme « compliqué » pour qualifier ces mêmes vocables, vocables qu'il a identifiés mais qu'il ne parvient pas à reproduire :

(19) il emploie les mots assez compliqués « coordonnées ", « je suis monamément », il avait dit quoi déjà ? «je suis modamément absent», il utilise des mots, au lieu de dire «j(e) suis absent » voilà (Antoine, 11 ans, inter + ).

Comme on peut le constater aussi dans les déclarations ci-dessous, les termes « coordonnées », « ultérieurement», " momentanément» et « recontacterai » ont en effet été remarqués par une dizaine d'enfants comme éléments saillants du style surveillé :

(20) au $1^{\mathrm{er}}$ il demande de laisser les coordonnées et au $2^{\mathrm{ème}}$ il dit pas les coordonnées (Tiphaine, 11 ans, inter-).

(21) dans la 1ère il y a des mots compliqués, comme « ultérieurement » (Laura, 10 ans, inter-).

(22) le 1er il dit « coordonnées » et « rencontaterai » (Gabriel, 10 ans, défav).

Bien sûr, à l'autre pôle du continuum, les expressions peu surveillées comme « les gars » et « lâchezvous », ont été remarquées par de nombreux enfants (80 ont relevé le terme "gars », 28 ont repris l'expression «lâchez-vous»), et ont souvent servi de point d'appui pour initier la discussion sur la variation. La formule «à plus » a également été notée, moins souvent mais à quelques reprises, comme chez Pierre :

(23) la 3ème c'est familier, comme «salut à plus » on met pas trop ça dans une conversation si on veut être des gens très polis (Pierre, 10 ans, fav). 
Il semblerait donc que certains traits saillants, en particulier lexicaux ${ }^{3}$, soient des éléments pivots de la construction du jugement sur les styles. Ces traits marquants semblent suffire, avec un effet de halo (Moreau \& Brichard, 1997), à catégoriser un énoncé dans une variété, ou tout au moins permettent à l'enfant de mettre des mots sur ce qu'il ressent, même si son impression se fonde sur d'autres éléments moins facilement conscientisés et verbalisables.

Néanmoins, si la saillance seule était en jeu dans les représentations, il serait difficile d'expliquer le phénomène suivant : certains enfants ne citent pas que des éléments réellement entendus mais aussi des traits absents des énoncés du document audio, dans un processus que l'on pourrait qualifier de « restauration stylistique $»^{4}$.

Il est également remarquable de constater que ces traits sont parfaitement cohérents avec le style concerné. Par exemple, alors qu'il n'y a pas de «ne » de négation dans les extraits présentés aux élèves, Margaux, comme neuf enfants de l'échantillon, emploie la négation à deux éléments pour référer au style surveillé :

(24) la première il dit pas des choses comme ça, il dit « désolé je ne suis pas là » (Margaux, 9 ans, $f a v)$.

Fabien, qui par ailleurs ne parvient pas à expliquer son jugement et n'a pas fait de commentaire explicite sur la variation, rétablit lui aussi une négation double là où elle est absente :

(25) il dit « salut les gars laissez-moi un message », « bonjour je ne suis pas là pour le moment laissez un message » (Fabien, 10 ans, défav).

Yanis reprend également une formulation surveillée en ajoutant le " ne » de négation, et semble ancrer son jugement dans une représentation stéréotypique des usages formels qu'il identifie au parler de son père en contexte professionnel :

(26) c'est sur un répondeur, c'est un monsieur. à un moment il dit « lâchez-vous les gars laissezmoi des messages », c'est pas pareil parce que l'autre il dit «bonjour je ne suis pas là laissez-moi un message ». il parle à des gens, enfin des gens par exemple je sais pas, si c'est un électricien par exemple. mon papa il parle comme ça (Yanis, 9 ans, inter + ).

Seynabou utilise en outre une structure syntaxique surveillée à l'oral (la coordination avec «car»), pourtant inexistante dans le document de départ; elle cherche par ailleurs à caractériser les styles avec la terminologie apprise à l'école :

(27) c'est un répondeur, c'est un monsieur qui parle, il utilise plusieurs moyens de langage, il parle à ses copains c'est le langage familier, le ler c'était quand il dit « vous pouvez me laisser un message car je ne suis pas là » apposé je crois (Seynabou, 10 ans, fav).

De la même manière, Kévin ajoute un «oui », forme d'acquiescement surveillée à l'oral, ainsi qu'un impératif typique des annonces de répondeurs standard :

(28) avec ses copains il dit « salut les potes » et tout, sinon « oui j(e) suis pas là veuillez laisser un message ». au début c'est pour sa famille, il dit gentiment (Kévin, 10 ans, défav).

Comme l'illustre l'extrait précédent, le même phénomène se produit pour le style le moins surveillé, des reprises à l'esprit mais pas à la lettre pouvant être relevées autour de choix syntaxiques proches de l'énoncé original, comme avec le terme «potes» remplaçant celui de «gars». En écho au « oui» surveillé de Kévin, on trouve aussi l'ajout de la forme non surveillée « ouais » chez Pauline, qui prend un ton très détendu pour imiter le troisième énoncé :

(29) le dernier il fait "ouais j(e) suis pas là na na na" et tout, comme ça (Pauline, 10 ans, inter-). 
On trouve également des ajouts prototypiques d'un style au niveau pragmatique, comme chez Alexandre, qui ne fait pas de commentaire métalinguistique sur la variation, mais qui cite les extraits en enrichissant l'énoncé le moins surveillé d'un marqueur discursif :

(30) il y avait un monsieur il était en train de laisser des messages. par exemple il disait «ah salut » sinon il disait... enfin il disait toujours "au revoir" après (Alexandre, 10 ans, inter + ).

Ces différents exemples illustrent donc selon nous une double tendance, à l'identification de traits saillants, essentiellement lexicaux et prosodiques d'une part, et à la reconstruction stéréotypique des énoncés d'autre part, reconstruction visible à travers l'usage cohérent en contexte de marques pourtant absentes de l'énoncé initial. Aussi, comme nous l'avons vu au paragraphe 1.3, la saillance et le stéréotype ne sont pas exclusifs l'un de l'autre, et les attitudes paraissent bel et bien se construire autour d'une imbrication des deux modalités représentationnelles.

La prédominance des références au lexique sur les autres niveaux linguistiques pourrait être due à un écart dans le degré d'accessibilité consciente aux phénomènes. En effet, l'enfant percevrait un ensemble d'éléments, à la fois lexicaux, morphosyntaxiques, pragmatiques, phonologiques et prosodiques, mais ne serait en mesure d'expliciter verbalement que certains d'entre eux, en l'occurrence les éléments lexicaux, les plus facilement identifiables et disponibles. Les autres niveaux ne sont pas ignorés : les enfants en perçoivent des traits caractéristiques, les intègrent, sont capables d'en restituer certains, voire de les produire sans les avoir entendus en les reconstruisant d'après leurs schémas représentationnels, mais ne sont pas encore en mesure de faire un commentaire à proprement parler métapragmatique à leur propos. C'est pourquoi des marques syntaxiques telles qu'une conjonction de coordination rare à l'oral ou la double négation peuvent être attestées dans les reformulations des enfants, sans qu'elles fassent pour autant l'objet de références explicites.

En outre, étant donné qu'un énoncé comprend un ensemble de traits plus ou moins congruents, dont certains seront plus saillants au niveau perceptif que d'autres dans certains contextes, et dont l'appréhension globale constituera le point d'appui des jugements des locuteurs, on peut formuler l'hypothèse selon laquelle la récurrence en réception de certains groupes d'éléments entendus fréquemment ensemble permettrait à l'enfant de se construire une sorte de schéma mental du style, autrement dit de s'en faire une représentation stéréotypique approximative globale. En effet, les variations entendues dans l'input ne sont pas aléatoires et l'enfant perçoit, dans certains énoncés spécifiques produits dans des situations données, des marques plus ou moins cohérentes entre elles, qui sont plus fréquemment produites ensemble que séparément. Par exemple, l'enfant est sans doute exposé plus souvent à des énoncés combinant, dans le même temps, des éléments de lexique informel, des traits syntaxiques spécifiques de l'oral informel, et des phénomènes phonologiques caractéristiques d'une surveillance articulatoire faible.

Les représentations sur le style se fonderaient donc sur des schémas stéréotypiques semi-conscients, mobilisés, élaborés et modifiés en hélice au gré des interactions rencontrées, à partir de la perception d'ensembles de traits congruents fréquemment produits ensemble dans l'input. La perception d'un trait unique, central dans un schéma, suffirait alors à activer un ensemble de traits prototypiques du style, quitte à restaurer stylistiquement certains traits absents de l'énoncé produit. Quant au jugement explicite sur les styles, qui suppose une capacité à produire un discours métalinguistique sur le phénomène, il s'exprimerait à travers un ensemble plus restreint de traits saillants emblématiques des styles conscientisés comme des catégories nommables et dénombrables. Ces jugements métapragmatiques reprennent ainsi en écho, en les simplifiant, des schémas représentationnels complexes et multi-niveaux.

\section{Synthèse des résultats et discussion}

Au final, cette enquête auprès d'enfants de 9 à 11 ans nous a permis de mieux comprendre le processus représentationnel, et de mieux connaître les critères sur lesquels les enfants fondent leurs jugements sur le style et comment ils s'actualisent dans l'élaboration des discours métapragmatiques sur la variation. 
Nos analyses montrent que plus de $60 \%$ des enfants de $9 / 11$ ans sont capables de proposer spontanément un commentaire de type métapragmatique sur la variation qui leur est proposée, et invoquent pour ce faire des critères relatifs au statut social du locuteur, à la norme, ou encore aux caractéristiques de l'interaction.

Notons en outre que les analyses plus qualitatives des réponses spontanées révèlent certains fonctionnements complexes de la construction des représentations (cf. figure 2). Les enfants prendraient appui sur certains traits saillants des styles, souvent prosodiques et lexicaux, qui activeraient des schémas cognitifs à partir desquels les enfants attribueraient une valeur stylistique aux productions. Par un double effet de halo et de restauration stylistique, l'ensemble de l'énoncé se verrait alors catégorisé dans un style, sorte de reconstruction stéréotypique homogène d'une réalité linguistique hétérogène.

Comme l'illustre la figure 2, l'actualisation du stéréotype influerait en retour sur la saillance des éléments perçus. Notons que le processus dans son ensemble ne comporte pas nécessairement que des volets conscients, certaines étapes pouvant relever de la dimension épipragmatique. En revanche, la restauration stylistique et la verbalisation du métadiscours semblent relever davantage de la conscience métapragmatique (en gris sur le schéma), cette capacité à mettre en mots la variation perçue étant ellemême un facteur d'actualisation des schémas.

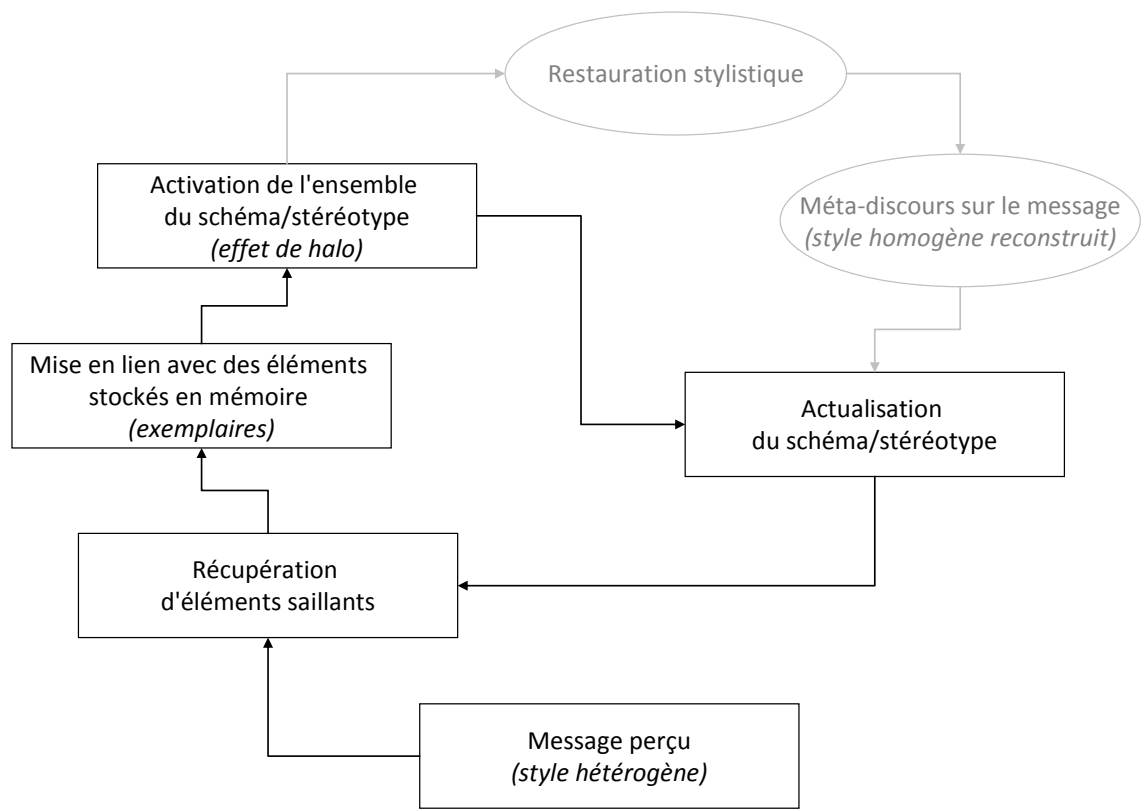

Figure 2 : Proposition de schématisation pour le processus de construction des représentations de la variation stylistique

En définitive, les capacités métapragmatiques des enfants s'appuient sur des processus évaluatifs complexes mettant en jeu la perception de variables plus ou moins saillantes et l'actualisation de représentations sociales en cours de construction. La saillance et les stéréotypes sociaux, qui sous-tendent toute la dynamique d'évaluation des styles et par conséquent la capacité à produire en retour un message approprié au contexte, s'avèrent être deux phénomènes fortement interdépendants. Ce constat permet d'avancer l'hypothèse que la dynamique de développement de la compétence de réception est largement tributaire du milieu et du réseau social dans lequel évolue l'enfant, lien qui reste à être exploré plus avant. Cette problématique s'inscrit alors dans des recherches nécessairement interdisciplinaires où seront pris en compte les processus cognitifs, les aspects sociaux, et la dynamique développementale. 


\section{Références bibliographiques}

Bates, E. (1976). Language and context. The acquisition of pragmatics. New York, San Francisco, London: Academic Press.

Bates, E. \& Macwhinney, B. (1979). A functionalist approach to the acquisition of grammar. In Ochs, E. \& Schieffelin, B. B. (Eds.), Developmental pragmatics. New York: Academic Press, 167-211.

Buson, L. (2009). Variation stylistique entre 5 et 11 ans et réseaux de socialisation scolaire : usages, représentations, acquisition et prise en compte éducative. Thèse de doctorat, Université Stendhal, Grenoble.

Campbell-Kibler, K. (2009). The nature of sociolinguistic perception. Language Variation and Change, 21, 135-156.

Clark, E. V. (1978). Awareness of language: some evidence from what children say and do. In Sinclair, A., Jarvella, R. J. \& Levelt, W. J. M. (Eds.), The child's conception of language. Berlin, New York: Springer-Verlag, 17-43.

Foulkes, P. \& Docherty, G. (2006). The social life of phonetics and phonology. Journal of Phonetics, 34, (4), 409438.

Fuchs, C. (1999). Diversity in linguistic representations. A challenge for cognition. In Fuchs, C. \& Robert, S. (Eds.), Language diversity and cognitive representations. Amsterdam, Philadelphia: John Benjamins, 3-19.

Gal, S. \& Irvine, J. (1995). The boundaries of languages and disciplines: how ideologies construct difference. Social research, 62, 967-1001.

Gombert, J. É. (1990). Le développement métalinguistique. Paris: PUF.

Irvine, J. (2001). "Style" as distinctiveness: the culture and ideology of linguistic differentiation. In Eckert, P. \& Rickford, J. R. (Eds.), Style and sociolinguistic variation. Cambridge: Cambridge University Press, 21-43.

Irvine, J. \& Gal, S. (2000). Language ideology and linguistic differentiation. In Kroskrity, P. (Ed.), Regimes of Language (pp.35-84). Santa Fe: School of American Research.

Kerswill, P. \& Williams, A. (2002). "Salience" as an explanatory factor in language change: evidence from dialect levelling in urban England. In Jones, M. C. (Ed.), Language Change. Amsterdam: Rodopi, 81-110.

Kristiansen, G. (2003). How to do things with allophones: linguistic stereotypes as cognitive reference points in social cognition. In Dirven, R., Pütz, M. \& Frank, R. M. (Eds.), Cognitive Models in Language and Thought. Ideology, Metaphors, and Meanings. Berlin/New York: Mouton de Gruyter, 69-120.

Kristiansen, G. (2008). Style-shifting and shifting styles: a socio-cognitive approach to lectal variation. In Kristiansen, G. \& Dirven, R. (Eds.), Cognitive sociolinguistics. Language variation, cultural models, social systems. Berlin, New York: Mouton de Gruyter, 45-90.

Landragin, F. (2004). Saillance physique et saillance cognitive. CORELA, 2, (2), http://corela.edel.univpoitiers.fr/document.php?id=142. Consulté le 3 juin 2009.

Landragin, F. (2005). Modélisation de la saillance visuelle et linguistique. Sixième Colloque des Jeunes Chercheurs en Sciences Cognitives (CJCSC'05), Bordeaux, 157-162.

Levelt, W. J. M., Sinclair, A. \& Jarvella, R. J. (1978). Causes and functions of linguistic awareness in language acquisition: some introductory remarks. In Sinclair, A., Jarvella, R. J. \& Levelt, W. J. M. (Eds.), The child's conception of language. Berlin, New York: Springer-Verlag, 1-14.

Lodge, A. (2007). Les Sarcelades de Nicolas Jouin (1684-1757). Langage et société, 121-122, (3-4), 113-128.

Louvet, E. (2005). Introduction à la psychologie sociale - Cours en ligne: http://psychologie.ustrasbg.fr/documentation/ELouvet/polycopie_L1_2005-2006.pdf. Consulté le 31 mars 2009.

Michinov, E. \& Monteil, J.-M. (2003). Attraction personnelle et attraction sociale : lorsque la saillance catégorielle annule la relation similitude-attraction. Canadian journal of behavioural science, 4, (35), 305-315.

Moreau, M.-L. \& Brichard, H. (1997). La part de la subjectivité dans l'évaluation de la qualité linguistique : une étude en Belgique francophone. Parole, 2, 121-145.

Schilling-Estes, N. (2002). Investigating stylistic variation. In Chambers, J. K., Trudgill, P. \& Schilling-Estes, N. (Eds.), The handbook of language variation and change. Oxford: Blackwell, 375-401. 
Slobin, I. (1978). A case study of early language awareness. In Sinclair, A., Jarvella, R. J. \& Levelt, W. J. M. (Eds.), The child's conception of language. Berlin, New York: Springer-Verlag, 45-54.

Smith, E. R. \& Zarate, M. A. (1992). Exemplar-based model of social judgment. Psychological Review, 99, (1), 3-21. Trudgill, P. (1986). Dialects in Contact. Oxford: Blackwell.

Warren, R. M. (1970). Perceptual restauration of missing speech sounds. Science, 167, 392-393.

${ }^{1}$ Cohérente en interne sur les éléments constituant le style, et en externe entre le style et les stéréotypes attribués au locuteur.

${ }^{2}$ Désormais défav, inter-, inter + , fav.

${ }^{3}$ Faute de transcription suffisamment fine à ce niveau, nous n'avons pas analysé les aspects prosodiques en détail mais avons remarqué que certains enfants s'en servaient aussi de point d'ancrage pour leur réflexion, en imitant la courbe intonative ou le ton spécifique à l'un des styles.

${ }^{4}$ Cette formulation établit un parallèle avec le phénomène de restauration phonémique décrit par Warren (1970), c'est-à-dire l'illusion qui consiste à entendre des sons qui ont en fait été supprimés du signal et remplacés par du bruit blanc. 